\title{
Moore-Federman syndrome and acromicric dysplasia: are they the same entity?
}

\author{
R M WINTER*, M A PATTON†, J CHALLENER \\ M BARAITSER\| \\ From * the Kennedy Galton Centre, Northwick Park Hospital; †St George’s Hospital, London; $¥$ Hinching. \\ brooke Hospital, Cambs; §The General Infirmary at Leeds; and |Institute of Child Health, London.
}

SUMMARY Four unrelated patients are reported with short stature, stiffness of the joints, short fingers, inability to make a fist, and thickened skin on the forearms. Investigations have failed to show a lysosomal storage disorder and radiographs show non-specific changes with a delayed carpal bone age. The clinical features in the four children are very similar to the recently described acromicric dysplasia. There are also similarities to Moore-Federman syndrome which has only been described in one family. The case is made that acromicric dysplasia and MooreFederman syndrome are the same entity.

Over the past seven years we have seen four patients with marked short stature, brachydactyly, limitation of movement of the joints, particularly in the hands, and mildly dysmorphic facies. The differential diagnosis has been between Moore-Federman dwarfism, ${ }^{1}$ geleophysic dysplasia, ${ }^{2}$ and the more recently described acromicric dysplasia. ${ }^{3}$ Here the four cases are described and the differential diagnosis discussed.

\section{Case reports}

CASE 1

This male infant was born at term by normal

Received for publication 12 July 1988.

Revised version accepted for publication 17 October 1988. delivery. Birth weight was $2900 \mathrm{~g}$. Both parents and his sister are normal. Nystagmus and short stature were noted at birth and vomiting was a problem up to the age of one year before it resolved. A cardiac murmur was detected which was thought to result from a small ventricular septal defect. The liver was slightly enlarged in the first year of life and the spleen has always been just palpable. Upper respira- $\overrightarrow{\vec{O}}$ tory tract infections have been associated with recurrent stridor. Laryngoscopy and tracheoscopy were carried out and this showed thickened supraglottic tissues; however, the cause of the stridor was not found. Height has always been well below the 3 rd centile; at the age of two years two months it was recorded as $76.8 \mathrm{~cm}$ and at six years nine months $97.7 \mathrm{~cm}$. Intelligence is normal.

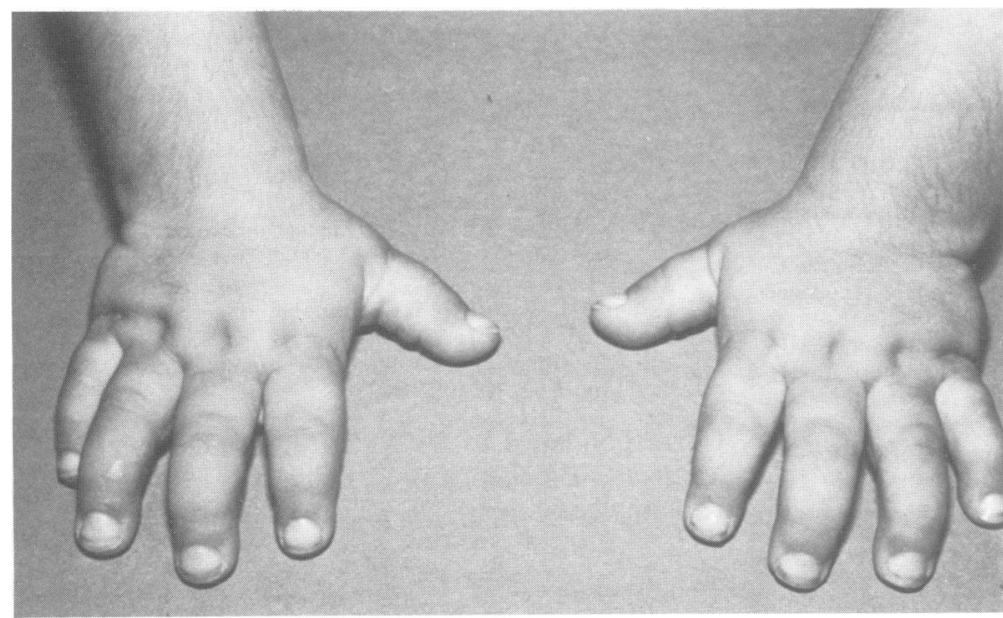

FIG 1 Hands of case 1 aged nine years. 
Examination at nine years showed a boy with marked proportionate short stature, short, stubby, wide fingers (fig 1), camptodactyly, and limitation in the range of movements of most joints. His face was round with narrow palpebral fissures, a broad nasal bridge, prominent epicanthic folds, and a short nose. Nystagmus was present and he was hypermetropic. The cardiovascular system was normal but there was mild hepatosplenomegaly.

Normal investigations included growth hormone and somatomedin studies, thyroid function and pituitary gonadatrophins, banded chromosome analysis, and repeated screening tests for mucopolysacchariduria and oligosacchariduria.

A skeletal survey showed mild abnormalities which included flat acetabular angles with well marked sacrosciatic notches, some fragmentation of both femoral head epiphyses with secondary change of the metaphyses, and mild angulation of the lower tibiae. The bone age was delayed by about two to

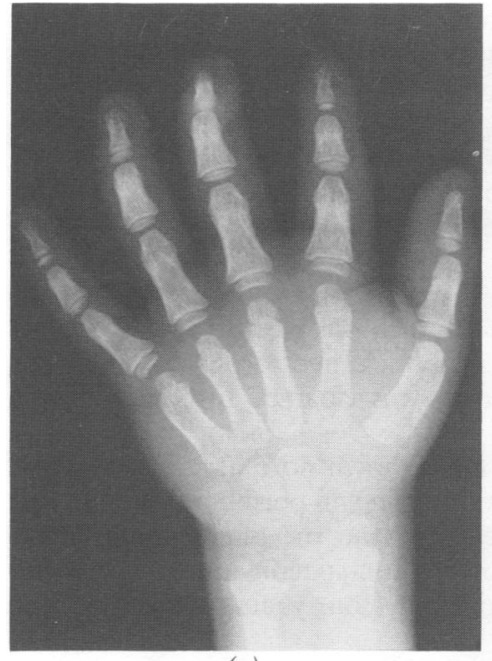

(a)

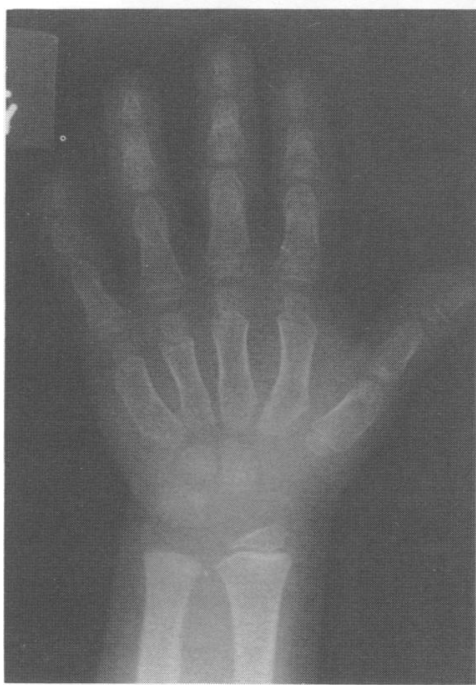

(c)

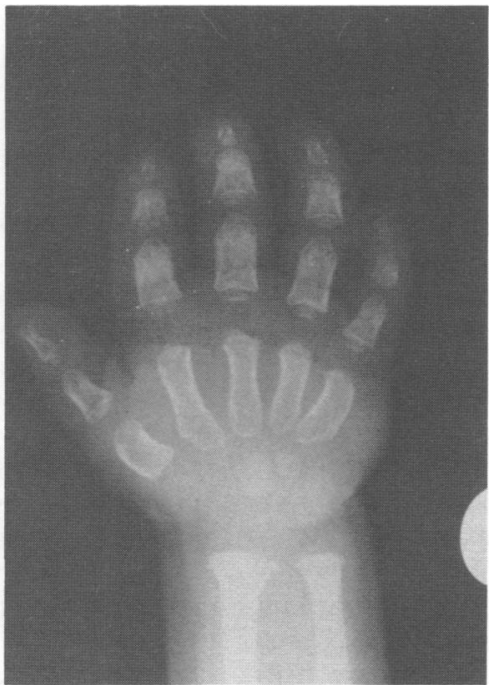

(b)

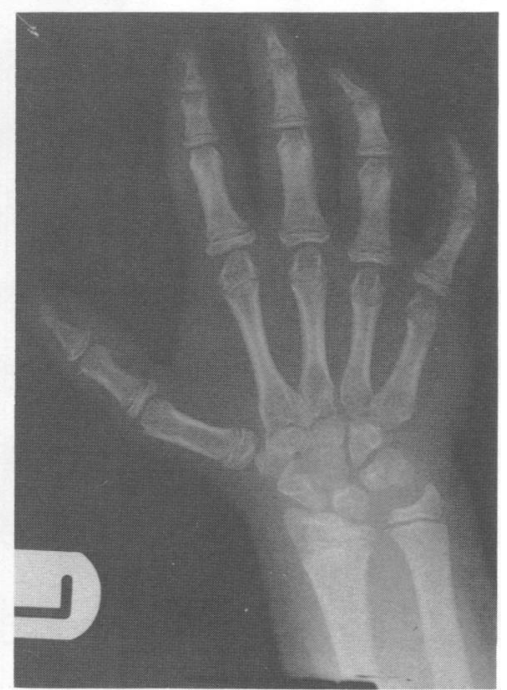

(d)
FIG 2 Hand radiographs of

(a) case 1 at six and a half years,

(b) case 2 at six and a half years,

(c) case 3 at seven and a half years,

(d) case 4 at 12 years. 
three years at the age of seven years (fig 2a). A biopsy of the epiglottis showed only mild chronic inflammation.

\section{CASE 2}

This male infant was delivered after a normal term pregnancy, weighing $2610 \mathrm{~g}$. There were no problems in pregnancy or the early neonatal period. The parents are normal Caucasians and unrelated.

The patient presented at the age of two years with proportionate short stature. His height was well below the $3 \mathrm{rd}$ centile $(74 \mathrm{~cm})$, crown-rump length $45 \mathrm{~cm}$, span $82 \mathrm{~cm}$, weight $10.3 \mathrm{~kg}$, and head circumference $50 \mathrm{~cm}$. He had unusual facies with bilateral ptosis, a broad, flat nose, and a long philtrum (fig 3). He had extremely short, pudgy fingers and could not manage to oppose his thumb across his palm. He was a little late to walk at 18 months when he tended to adopt a tiptoe walking posture. Ophthalmological examination was normal. He has been followed regularly and has continued to grow well below but parallel to the 3rd centile for height and just below and parallel to the 3rd centile for weight. His development has been normal apart from some hearing problems principally related to dense occlusive wax and hairiness of abnormally small meati. He has developed some joint stiffness, particularly in the left arm, spine, hips, and knees, and now has a hoarse voice and thickened skin. An echocardiographic examination was normal.

Investigations have included normal tests for mucopolysacchariduria and oligosacchariduria, and normal lysomal enzymes ( $\beta$-galactosidase, $\alpha$ fucosidase, $\alpha$-mannosidase, neuraminidase, $\beta$ glucuronidase, I cell screen). Radiographs showed a markedly delayed bone age (nine months at a chronological age of two years; two years at a chronological age of six years), and a suggestion of proximal tapering of the metacarpals (fig $2 b$ ).

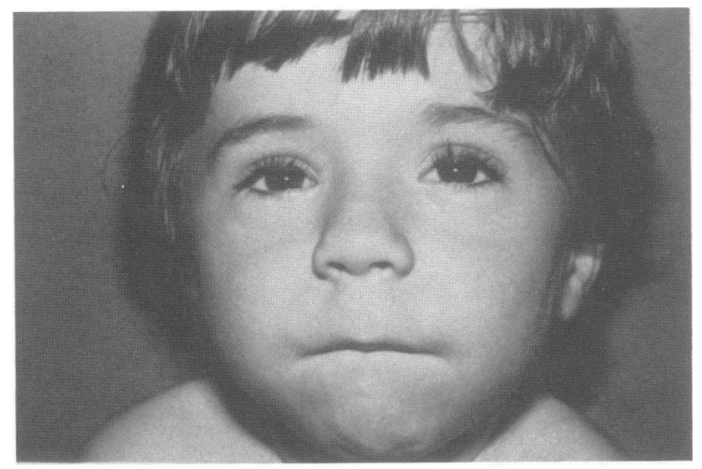

FIG 3 Facial features of case 2 at four years.
CASE 3

This boy was born at term by normal delivery, birth weight $3714 \mathrm{~g}$. Both parents and his sister are normal. He has always been short with a height of $104 \mathrm{~cm}$ at seven and a half years and $114 \mathrm{~cm}$ at 10 years, both well below the 3rd centile. His other problems have been restriction of joint movement, signs of carpal tunnel syndrome, and marked hypermetropia. In addition he has suffered from hay fever and allergies to penicillin and aspirin. During an anaesthetic he developed pyrexia and sustained muscle contracture after a dose of suxamethonium. Both parents underwent muscle biopsy and screening for malignant hyperpyrexia, but these tests were found to be negative. The proband was thought at the time to be too young to undergo these tests.

On examination at 10 years he was proportionately short with a round face, narrow palpebral fissures, and hypermetropia (fig 4). There was limitation of movements of most joints including extension of the elbow and supination and pronation of the forearms. The fingers were short with mild contractures and he was unable to make a complete fist (fig 5). The thumbs were short, stubby, and broad. The skin was thickened, especially over the forearms. His voice was hoarse. Intelligence was normal.

Investigations have shown normal chromosomes, no vacuolated lymphocytes in peripheral blood, and no mucopolysacchariduria or oligosacchariduria. Radiographs showed non-specific limb shortening with a delayed bone age (four years seven months at

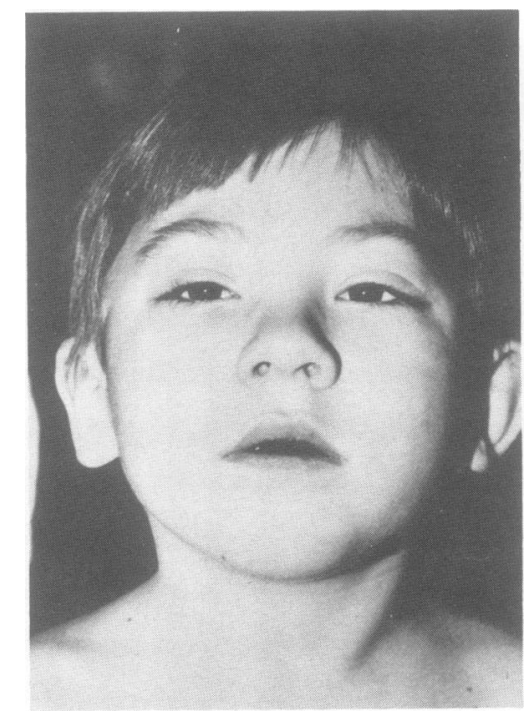

FIG 4 Facial features of case 3 at 10 years. 


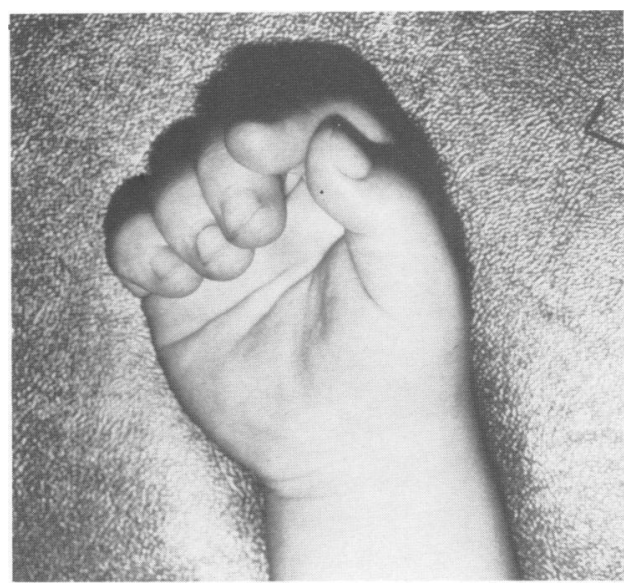

FIG 5 Maximum degree of finger flexion, case 3.

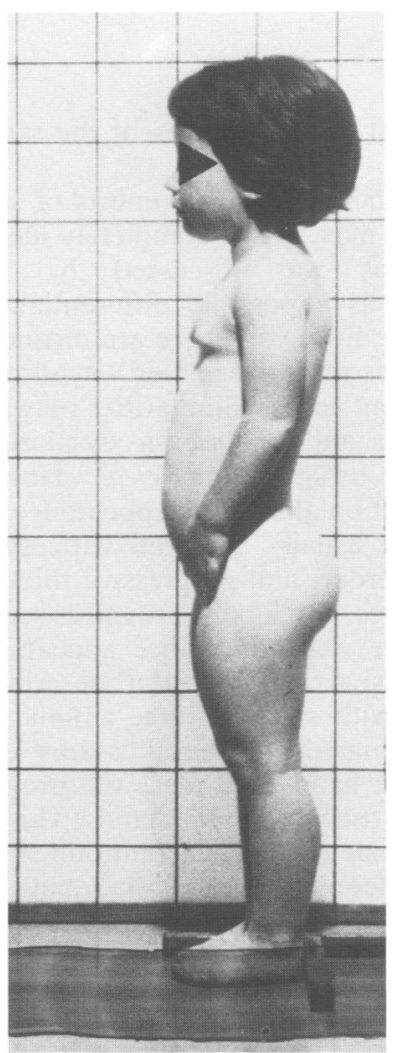

FIG 6 Case 4 aged 111/2 years. a chronological age of seven years five months) (fig 2c).

\section{CASE 4}

This girl was born of Caucasian parents by normal delivery at term, birth weight $2636 \mathrm{~g}$. There is one normal brother. Poor weight gain was noted in infancy and coeliac disease was excluded at one year. There was concern about proportionate short stature from the first year of life and since then she has continually been below the 3 rd centile for height $(70.5 \mathrm{~cm}$ at two years; $90 \mathrm{~cm}$ at six years; $114 \mathrm{~cm}$ at $11 \frac{1 / 2}{2}$ years). From four years there has been limitation of finger flexion. Intelligence is normal.

On examination at $11 \frac{1}{2}$ years there was limitation of movements of many joints including restriction of extension of the elbows and supination of the forearms, and mild contractures of the fingers with an inability to make a fist. The fingers were short and stubby and there was an exaggerated lumbar lordosis (fig 6). She was hypermetropic and was unable to look upwards. This was thought to be because of Brown's superior tendon sheath syndrome. The face was round and there was a mild upward slant to the narrow palpebral fissures. Investigations showed normal chromosomes and negative tests for mucopolysacchariduria and oligosacchariduria. Radiographs showed a bone age delayed by two to three years at the age of 11 years, but no other specific changes (fig $2 \mathrm{~d}$ ).

\section{Discussion}

The original diagnosis in all four patients described here was Moore-Federman dwarfism. This autosomal dominant syndrome was described in one family in $1965 .^{1}$ The main features were short stature, hypermetropia, asthma, and stiff joints especially in the hands. Those affected were unable to make a fist. No details were given about facial features, and the hands did not appear from the photographs to be markedly shortened. The MooreFederman syndrome is one of those rare conditions which has the distinction of having been 'dropped' from the third edition of Smith's Recognizable patterns of human malformation. ${ }^{4}$ Since the original description, there have been no further convincing case reports, but Maroteaux et $a l^{3}$ described a very similar condition in six isolated cases, which they called acromicric dysplasia. The main features are short stature, short hands and feet, and a characteristic facial appearance. The latter consists of narrowness of the palpebral fissures and a short stubby nose with anteverted nostrils. Radiography of the hands shows short, stubby metacarpals and phalanges and 
TABLE Moore-Federman syndrome, acromicric dysplasia, and current cases: comparison of clinical features.

\begin{tabular}{|c|c|c|c|c|c|c|}
\hline & \multirow{2}{*}{$\begin{array}{l}\text { Moore-Federman } \\
\text { dwarfism }\end{array}$} & \multirow{2}{*}{$\begin{array}{l}\text { Acromicric } \\
\text { dysplasia* }\end{array}$} & \multicolumn{4}{|c|}{ Present cases } \\
\hline & & & 1 & 2 & 3 & 4 \\
\hline Short stature & $6 / 6$ & + & + & + & + & + \\
\hline \multicolumn{7}{|l|}{ Skeletal } \\
\hline Generalised stiff joints & $6 / 6$ & - & + & + & + & + \\
\hline Inability to make fist & $5 / 6$ & $+1-$ & + & + & + & + \\
\hline \multicolumn{7}{|l|}{ Eyes } \\
\hline Hypermetropia & $5 / 6$ & - & + & - & + & + \\
\hline Glaucoma & $1 / 6$ & - & - & - & - & - \\
\hline Cataract & $1 / 6$ & - & - & - & - & - \\
\hline Retinal detachment & $1 / 6$ & - & - & - & - & - \\
\hline \multicolumn{7}{|l|}{ Facies } \\
\hline Round face & $?$ & $+1-$ & + & + & + & + \\
\hline Narrow palpebral fissures & $?$ & + & + & + & + & + \\
\hline Small/short nose & $?$ & + & + & + & + & + \\
\hline \multicolumn{7}{|l|}{ Other } \\
\hline Thickened skin & $5 / 6$ & - & $?$ & + & + & + \\
\hline Hepato/splenomegaly & $4 / 6$ & - & + & - & - & - \\
\hline Hoarse voice/stridor & $3 / 6$ & - & + & + & + & - \\
\hline Asthma/allergies & $4 / 6$ & - & + & - & + & - \\
\hline Cardiac anomalies & WPW(1) & $\operatorname{ASD}(1)$ & $\begin{array}{l}\text { Small } \\
\text { VSD }\end{array}$ & - & - & - \\
\hline
\end{tabular}

"From Maroteaux et $a l^{3}$; six patients, not described individually.

$\mathrm{WPW}=$ Wolff-Parkinson-White syndrome; $\mathrm{ASD}=$ atrioseptal defect; $V S D=$ ventriculoseptal defect.

pointing of the proximal portion of the last four metacarpals with a lateral and medial notch at the base of the second and fifth metacarpals respectively. The long bones are relatively normal apart from slight deformation of the femoral heads in some cases. Bone age in the wrists and hands is delayed.

Our four cases show many features in common with those described as having acromicric dysplasia. In particular, the short stature, short, stubby hands, and facial appearance seem to be very similar. Features present in the present cases but not in those of Maroteaux $e t a l^{3}$ include generalised joint stiffness, hypermetropia, thickened skin, and a hoarse voice or stridor. However, the latter cases are not described in great clinical detail. Maroteaux et $a l^{3}$ state that the radiological appearance of the hands in their cases was pathognomonic, but it is difficult to be entirely convinced by their illustrations. The radiological features in our cases showed similarities to those described in acromicric dysplasia, especially case 2 . Maroteaux et al, ${ }^{3}$ in a footnote to their paper, mentioned that they had seen an affected mother and son, suggesting autosomal or possibly $\mathrm{X}$ linked dominant inheritance. There are certainly similarities between the present cases, the autosomal dominant condition described by Moore and Federman, ${ }^{1}$ and the cases described by Maroteaux et $a l^{3}$ as acromicric dysplasia, and it is possible that they all represent the same condition (table).

Finally, there are other reports of similar conditions which may not be completely identical. Leri's pleonosteosis was discussed by Moore and Federman $^{1}$ as a possible differential diagnosis in their family; however, there are distinctive radiological changes reported in Leri's syndrome not seen in the present cases. ${ }^{5}$ Similarities between acromicric dysplasia and geleophysic dysplasia have been pointed out by Maroteaux et al. ${ }^{3}$ However, in the latter condition the facial appearance is different, the liver is usually enlarged with evidence of a storage disorder, and progressive thickening of the cardiac valves with a poor prognosis seems to occur. Furthermore, the inheritance appears to be autosomal recessive. ${ }^{2}$ Pfeiffer et al ${ }^{6}$ described a brother and sister with short stature, amimic (expressionless) facies, narrow palpebral fissures, enamel hypoplasia, short hands and feet, and progressive joint stiffness, particularly in the hands. The boy suffered 0 from aortic stenosis. This condition shows similarities to geleophysic dysplasia, but is probably different from the cases described here.

In summary, we describe four isolated cases with a syndrome showing great similarity to acromicric dysplasia. There is also considerable overlap with Moore-Federman dwarfism, and it is possible that these two conditions are identical. 
The authors would like to thank Dr P Husband for allowing them to report one of the cases.

\section{References}

${ }^{1}$ Moore WT, Federman DD. Familial dwarfism and 'stiff joints'. Arch Intern Med 1965;115:398-404.

2 Spranger J, Gilbert EF, Arya S, et al. Geleophysic dysplasia. Am J Med Genet 1984;19:487-99.

${ }^{3}$ Maroteaux P, Stanescu R, Stanescu V, et al. Acromicric dysplasia. Am J Med Genet 1986;24:447-59.

4 Smith DW. Recognizable patterns of human malformation. 3rd ed. Philadelphia: Saunders, 1982.
${ }^{5}$ Rukavina JG, Falls HF, Holt JF, Block WD. Levi's pleonosteosis: a study of a family with a review of the literature. $J$ Bone Joint Surg [Am] 1959;41:397-408.

6 Pfeiffer RA, Palm D, Teller W. A syndrome of short stature, amimic facies, enamel hypoplasia, slowly progressive stiffness of the joints, and a high-pitched voice in two siblings. $J$ Pediatr 1977;91:955-7.

Correspondence to $\operatorname{Dr} \mathbf{R} \mathbf{M}$ Winter, Kennedy Galton Centre, Northwick Park Hospital, Harrow, Middlesex HA1 3UJ. 